\title{
Organ Dysfunction After Surgery in Patients Treated With Individualized or Standard Blood Pressure Management
}

\author{
Luke J. Laffin, MD and Michael R. Laffin, MD \\ Section of Cardiology, University of Chicago Medicine, Chicago, Illinois (L. J. Laffin); Department \\ of Surgery, University of Alberta, Edmonton, Alberta, Canada (M. R. Laffin)
}

\section{To the Editor}

In a recent study, individualized perioperative blood pressure management comparedwith standard care in the setting of major surgery was found to reduce the risk of postoperative organ dysfunction. ${ }^{1}$ Although the study was hypothesis generating, the manner and rigor with which reference or normal resting blood pressure was obtained warrants further explanation.

Resting blood pressure from the preoperative anesthesiology consultation was used as the reference value and, if unavailable, the blood pressure measurement recorded on a surgical ward the day before surgery, while the patient was supine, was used. Clearly, these values may vary widely. Basing the intervention of keeping the SBP within $10 \mathrm{mmHg}$ of the normal resting value obtained with a nonuniform method of measurement may affect the internal validity of the results. Furthermore, international guidelines recognize that preoperative blood pressure management should be rigorous and evaluated in the context of historical blood pressures taken in a primary care setting. ${ }^{2}$ In this trial, in which greater than $65 \%$ of patients were taking an antihypertensive medication at baseline, such an approach would have been particularly valuable.

The data suggest that individualized blood pressure management can improve postoperative surgical outcomes. However, fundamental questions remain regarding the validity of the individualized therapeutic blood pressure targets. Were multiple blood pressure measurements taken in the preoperative clinic and averaged? Were these measurements obtained in the supine position? Was blood pressure obtained by a manual auscultatory method or via an oscillometric blood pressure machine? The answers to these questions will reveal the trust that should be placed in each patient's normal resting value and consequently the trust that should be placed in the trial's results. Reproducibility and accuracy when measuring blood pressure are critical.

Corresponding Author: Luke J. Laffin, MD, University of Chicago Medicine, 5841 S Maryland Ave, MC 6080, Chicago, IL 60637 (luke.laffin@uchospitals.edu).

Conflict of Interest Disclosures: The authors have completed and submitted the ICMJE Form for Disclosure of Potential Conflicts of Interest and none were reported. 


\section{References}

1Futier E, Lefrant JY, Guinot PG, et al. INPRESS Study Group. Effect of individualized vs standard blood pressure management strategies on postoperative organ dysfunction among high-risk patients undergoing major surgery: a randomized clinical trial. JAMA. 2017; 318(14):1346-1357. [PubMed: 28973220]

2Hartle A, McCormack T, Carlisle J, et al. The measurement of adult blood pressure and management of hypertension before elective surgery: joint guidelines from the Association of Anaesthetists of Great Britain and Ireland and the British Hypertension Society. Anaesthesia. 2016; 71(3):326-337. [PubMed: 26776052] 